\title{
Optical observations of supernova 2012aw
}

\author{
Subhash Bose ${ }^{1}$, Brijesh Kumar ${ }^{1}$, Firoza Sutaria ${ }^{2}$, Rupak Roy ${ }^{1}$, \\ Brajesh Kumar ${ }^{1}$, Vijay K. Bhatt ${ }^{1}$, Sayan Chakraborti ${ }^{3}$ \\ ${ }^{1}$ Aryabhatta Research Institute of Observational Sciences, Nainital, India \\ email: bose@aries.res.in; email@subhashbose.com \\ ${ }^{2}$ Indian Institute of Astrophysics, Bangalore, India \\ ${ }^{3}$ Institute for Theory and Computation, Harvard
}

\begin{abstract}
We present optical $U B V R I$ photometric and low-resolution spectroscopic follow-up observations of a type II SN 2012aw in a nearby ( 10 Mpc) galaxy M95 during 4 to 270 days post-explosion. The evolution characteristics of optical brightness and color are found to have striking similarity with the archetypal type IIP SN $1999 \mathrm{em}$. The mid-plateau $M_{V}$ is $-16.7 \mathrm{mag}$ and the ejected nickel mass is $\sim 0.06 \mathrm{M}_{\odot}$. The presence and evolution of optical spectral features during $7 \mathrm{~d}$ to $104 \mathrm{~d}$ are also similar to SN $1999 \mathrm{em}$ as well as other normal type IIP events. The mid-plateau photospheric velocity is around $4200 \mathrm{~km} \mathrm{~s}^{-1}$ which is same as that of SN 2004et at similar phases, indicating similar energy of explosion i.e. $2 \times 10^{51} \mathrm{erg} \mathrm{s}^{-1}$.
\end{abstract}

Keywords. (stars:) supernovae : individual (SN 2012aw); techniques: photometric, spectroscopic

\section{Observations}

The broadband photometric data in $U B V R I$ Johnson-Cousins system are collected using the 104-cm Sampurnanand Telescope which is operated by the Aryabhatta Research Institute of Observational Sciences, Nainital, India (Sagar 2006). The data ranges from 4d to $270 \mathrm{~d}$. The long-slit low-resolution $(\sim 10 \AA)$ spectra in the visible range $(4000-8000 \AA)$ were collected at 14 phases during $7 \mathrm{~d}$ to $270 \mathrm{~d}$ post explosion; nine from $2 \mathrm{~m}$ IUCAA Girawali Observatory Telescope, Pune and five from 2m Himalayan Chandra Telescope, Hanle. Data reduction is done using IRAF in standard manner.

\section{Preliminary results}

The $U B V R I$ light-curve of SN 2012aw is presented in Fig. 1. The plateau phase of about 100d duration is clearly visible and the light-curve shape matches well with SN $1999 \mathrm{em}$. The optical bolometric light-curves of other well studied SNe are also overplotted. A comparison of nebular phase light-curve with SNe 1999em and 2004et suggests that the ${ }^{56} \mathrm{Ni}$ produced in 2012aw are similar to these events. Comparing with SN 1987, we derive the mass of ${ }^{56} \mathrm{Ni}$ for $\mathrm{SN} 2012$ aw to be $\sim 0.058 \mathrm{M}_{\odot}$.

Fig. 2 [Left] shows low-resolution spectra at 14 phases between $7 \mathrm{~d}$ and 104d. Using the Na I D absorption dips and employing empirical relation from Poznanski, Prochaska, \& Bloom (2012), the total reddening $E(B-V)$ in direction to SN 2012aw has been estimated to be $\sim 0.075$ mag. The SYNOW modeling of spectra are done for all 14 spectra to identify lines and to determine photospheric velocities. Fig. 2 [Right] shows the SYNOW modeling for the phases $7 \mathrm{~d}$ and $61 \mathrm{~d}$ along with all identified spectral features. Striking similarity of spectral features with that of SN 1999em is noticed. The velocity profile of 

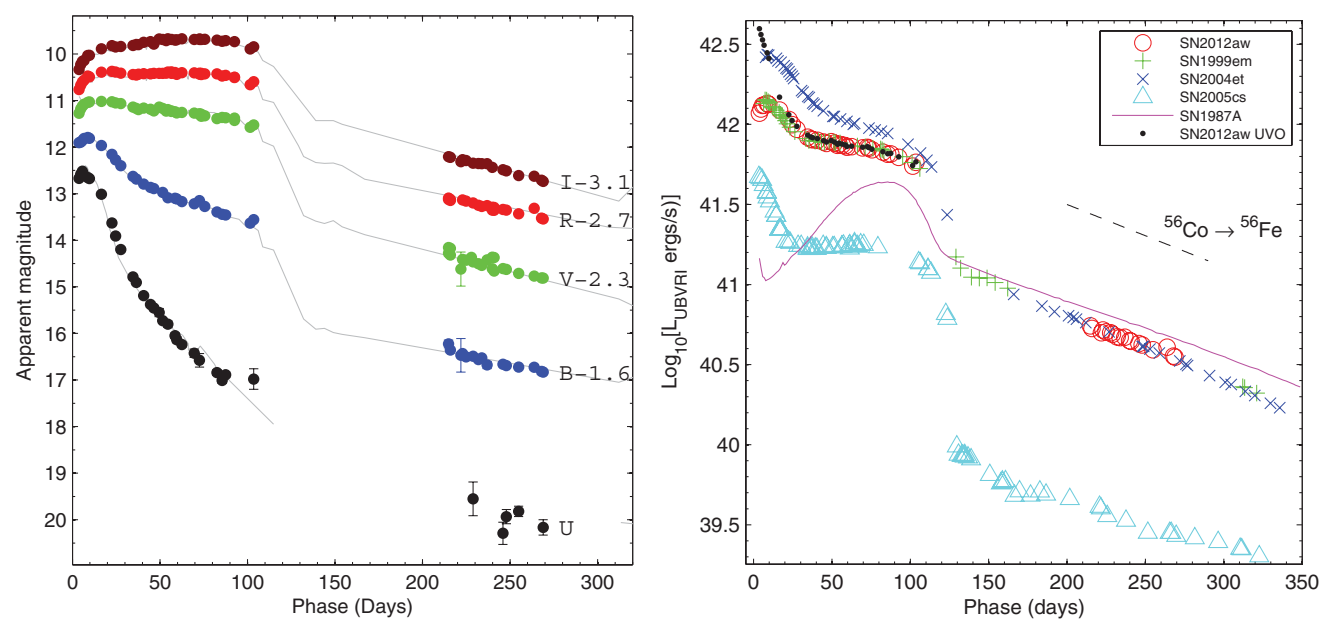

Figure 1. The apparent magnitude (left) and bolometric (right) light-curve of SN 2012aw. The apparent light curve of archetypal type IIP SN 1999em is shown in grey solid lines. The UV-optical bolometric curve for SN 2012aw is generated by incorporating UV data from Bayless et al. $(2012)$
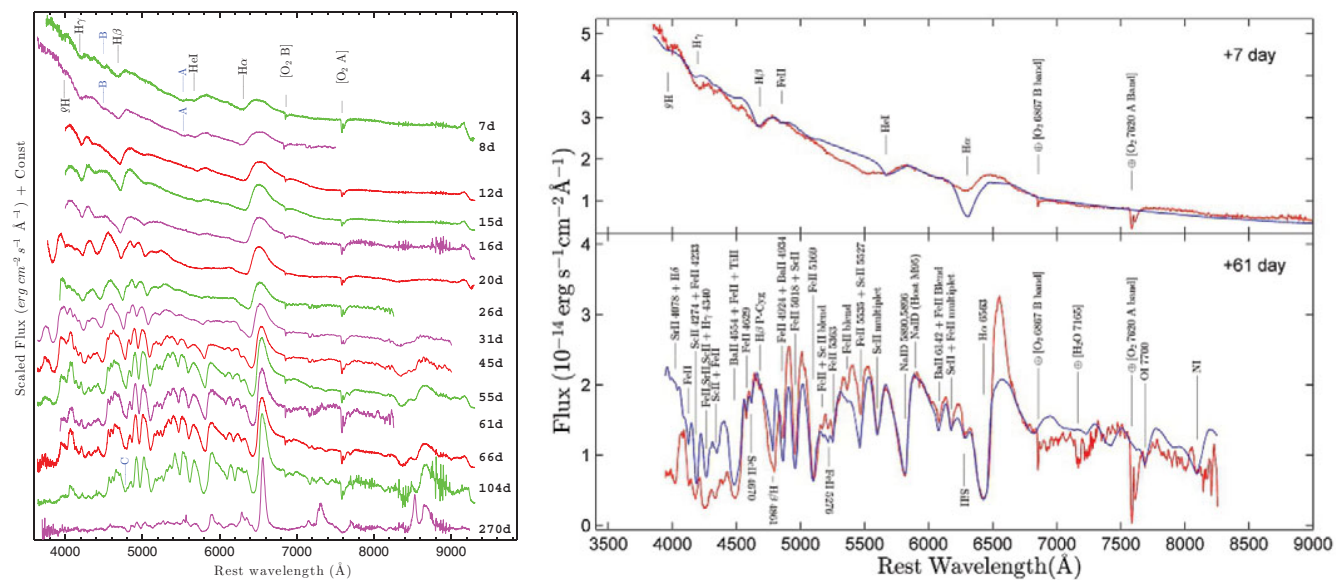

Figure 2. Left : The Doppler-corrected spectra of SN 2012aw are shown for 14 phases during $7 \mathrm{~d}$ to $270 \mathrm{~d}$. Right : The SYNOW modeling is shown for $7 \mathrm{~d}$ and $61 \mathrm{~d}$ spectrum. Model spectra are shown with thick solid line, while the observed ones are in thin solid line.

SN 2012aw estimated using Fe II lines $(4924,5018,5169 \AA)$ is found to be matching well with SN 2004et, though it is consistently higher than SN 1999em at all phases.

\section{References}

Bayless A. J., et al. 2012, arXiv, arXiv:1210.5496

Poznanski D., Prochaska J. X., \& Bloom J. S. 2012, MNRAS, 426, 1465

Sagar R. 2006, BASI, 34, 37 